
\title{
is Research Suare \\ Subconjunctival injections of triamcinolone acetonide to treat uveitic macular edema
}

\section{Yi Qu}

Peking Union Medical College Hospital

Xinshu Liu

the fourth people's Hospital in Shenyang

Anyi Liang

Peking Union Medical College Hospital

Chan Zhao

Peking Union Medical College Hospital

Fei Gao

Peking Union Medical College Hospital

Zhang Meifen ( $\nabla$ meifen_zhang@hotmail.com )

Peking Union Medical College Hospital https://orcid.org/0000-0002-7581-6800

\section{Research article}

Keywords: Triamcinolone acetonide, subconjunctival injection, uveitis, macular edema, intraocular pressure

Posted Date: March 9th, 2020

DOI: https://doi.org/10.21203/rs.2.16180/v2

License: (c) (1) This work is licensed under a Creative Commons Attribution 4.0 International License.

Read Full License 


\section{Abstract}

Background: To evaluate the efficacy and safety of subconjunctival triamcinolone acetonide (TA) injections for treating uveitic macular edema (UME).

Methods: This retrospective case series study included patients with UME who received subconjunctival TA injections with a minimum follow-up period of 6 months. The main outcome measure was central macular thickness. The secondary outcome measures included best corrected visual acuity, recurrence rate and intraocular pressure.

Results: In total, 68 patients (83 eyes) were enrolled in this study. The mean CMT decreased from $456.9 \pm$ $171.1 \mu \mathrm{m}$ at baseline to $324 \pm 175.9 \mu \mathrm{m}, 305.6 \mu \mathrm{m} \pm 147.7 \mu \mathrm{m}, 331.8 \pm 154.3 \mu \mathrm{m}$ and $281.1 \pm 147.6 \mu \mathrm{m}$ at $1-, 2-, 3-$ and 6 -months post injection, respectively (all $P<0.01$ ). A total of $21 / 83$ eyes exhibited elevated IOPs, of which 14 were controlled with topical IOP-lowering agents and 7 eyes underwent surgical removal of subconjunctival TA deposit.

Conclusion: Subconjunctival TA injections appear to be safe and effective for UME.

\section{Background}

Macular edema (ME) is one of the most common complications of uveitis which may result in visual impairment and even blindness ${ }^{1-4}$. The mechanism of ME is believed to result from fluid leakage across the blood-retinal barrier and fluid accumulation in the macular region, sometimes with a characteristic distribution in the outer plexiform layer and subretinal area ${ }^{1}$. While corticosteroids remain the first line treatment for uveitic macular edema (UME), immunosuppressants such as cyclosporine, methotrexate, azathioprine and mycophenolate mofetil are usually required for chronic and intractable UME. Sustainedrelease corticosteroid implants ${ }^{5}$, anti-VEGF agents and anti-TNF-a agents are recently emerged options for $\mathrm{UME}^{1,6}$.

Despite the advantages of the corticosteroid implants, triamcinolone acetonide (TA), a long-acting glucocorticoid, is still widely used for its efficacy and affordable cost ${ }^{7}$. While reports on periocular ${ }^{8}$ or intraocular injections of TA are numerous ${ }^{9-13}$, however, few studies have been conducted on subconjunctival injections of TA for treating $\mathrm{UME}^{14-16}$.

This study aimed to evaluate the efficacy and safety of subconjunctival TA injections in treating UME.

\section{Methods}

This study was a retrospective cases series of UME patients who underwent subconjunctival TA with regular follow up. Written informed consent was obtained from all participants before the subconjunctival TA injection(s) and any other invasive procedures/examinations. The study adhered to the ethical standards stated in the 1964 Declaration of Helsinki. 
Patient Eligibility and Exclusion Criteria

The clinical data of UME patients who received subconjunctival TA injections from January 2009 to December 2018 in the Department of Ophthalmology, Peking Union Medical College Hospital were collected and analyzed. The inclusion criteria were as follows: a) new onset of unilateral UME, or bilateral UME with unilateral aggravation, of any anatomical type (anterior, middle, posterior or pan uveitis); b) if the patients was on systemic corticosteroid, the dose should be $\leq 15 \mathrm{mg}$ prednisone or equivalent; $\mathrm{c}$ ) absence of significant ocular inflammation that require, or the patient refused or has contraindications for, initiation or up titration of systemic corticosteroids and/or immunosuppressants; d) had been regularly followed up to at least 6 months after the (last) subconjunctival TA injection. e) patients with complete clinical data at baseline and the 1-, 2-, 3- and 6-months post-injection visits. The exclusion criteria were as follows: a) history of any other ocular disease (e.g., diabetic retinopathy or retinal vascular obstruction) that may cause macular edema; b) received periocular or intraocular injections within 6 months before the (first) subconjunctival TA injection; c) presence or development of posterior synechia or media opacity such as cataract that compromise satisfactory fundus evaluation and quality of (optical coherence tomography) OCT images.

\section{Examination and Treatment Procedures}

The procedure was performed in the outpatient department. Patients received subconjunctival injections of TA in a supine position. To anesthetize the injected eye, a single application of $0.4 \%$ oxybuprocaine hydrochloride eye drops (Santen Pharmaceutical Co., Ltd.) was applied. $1 \mathrm{~mL}$ syringe contained 20mg Triamcinolone acetonide (Kunming Jida Pharmaceutical Co., Ltd., concentration: $40 \mathrm{mg} / \mathrm{mL}$ ) was injected into the inferior fornix, and the drug deposit could be seen under the conjunctiva. Patients were asked to monitor their eye pressure every 2 weeks after the intervene. Systemic corticosteroids or immunosuppressants were not initiated or up-titrated. Corticosteroid eye drops and topical NSAIDs were not used in any of the cases. While Topical IOP-lowering agents were applied as first line treatment for IOP elevation, such as beta-blockers, carbonic anhydrase inhibitors, and alpha-agonist. For patients with IOP over $30 \mathrm{mmHg}$ that cannot be controlled with topical eye drops, surgery to remove TA deposit is recommended

The main outcome measure was central macular thickness (CMT) measured by OCT. The secondary outcome measures included best corrected visual acuity (BCVA), recurrence rate and IOP within 6 months after the injection.

OCT Acquisition 
The CMT was measured using an Optovue OCT (Optovue, Fremont, CA) or 3D-OCT 2000 (Topcon Corporation, Japan) devices. In order to follow-up, the same device was applied for each patient. AutoRescan features were used to ensure that the follow-up scans matched the baseline.

Statistic Analysis

Statistic analysis was performed using IBM SPSS software, version 25.0 (IBM SPSS, USA). Visual acuity was obtained from each patient's medical records and converted to a logarithm of the minimal angle of resolution (logMAR) for statistic analysis. Paired t-tests were performed to analyze logMAR visual acuity and CMT. A P value $<0.05$ was considered significant.

\section{Results}

In this retrospective, observational case series study, 68 patients ( 17 males and 51 females, 83 eyes) were enrolled. The age of included patients ranged from 11 to 78 (49.2 \pm 14.1$)$ years; $38 / 68$ patients (55.88\%) received only one injection, while other patients received several injections in one eye or in both eyes. Of the 15 patients with both eyes included, none received bilateral subconjunctival TA injection simultaneously. The demographic features of patients at baseline were shown in Table 1.

The mean CMTs of the subconjunctival TA-injected eyes were significantly reduced. The mean CMT decreased from $456.9 \pm 171.1 \mu \mathrm{m}$ before the injection to $324 \pm 175.9 \mu \mathrm{m}(P<0.01), 305.6 \mu \mathrm{m} \pm 147.7 \mu \mathrm{m}$ $(P<0.01), 331.8 \pm 154.3 \mu \mathrm{m}(\mathrm{P}<0.01)$ and $281.1 \pm 147.6 \mu \mathrm{m}(\mathrm{P}<0.01)$ at $1-, 2-, 3-$ and 6 - months after injection, respectively (Figure 1 ). A total of $22 / 83$ eyes (26.51\%) relapsed within 6 months; $10 / 22$ eyes received a second injection and were still responsive.

BCVA increased from logMAR $0.5 \pm 0.3$ at baseline to logMAR $0.4 \pm 0.3(P<0.01), \log M A R 0.4 \pm 0.3$ $(P<0.01), \log M A R 0.4 \pm 0.4(P<0.01)$ and $\log M A R 0.4 \pm 0.3(P<0.01)$ at the 1-, 2-, 3- and 6-months postinjection visits, respectively. (Figure 2).

Elevation of IOP ( $\geq 21 \mathrm{mmHg}$ ) was observed in $21 / 83$ (25.30\%) eyes. Among them, $8 / 21$ (38.09\%), 6/21 (28.57\%) and 3/21 (14.29\%) eyes had peak IOPs between 21 and $25 \mathrm{mmHg}, 25$ and 30mmHg, 30 and $35 \mathrm{mmHg}$, respectively; and 4/21(19.05\%) eyes had peak IOPs over 35mmHg."14/21(66.67\%) eyes were well controlled by 1 or 2 kinds of topical IOP-lowering agents, while 7 eyes (33.33\%) underwent surgical removal of the subconjunctival TA deposit. Figure 3 was the Kaplan-Meier survival analysis of the patients with elevated IOP, which revealed that the time frame for IOP rise was the first 2 months after the injections.

\section{Discussion}


$M E$ is frequently encountered in patients with uveitis ${ }^{17,18}$ and may cause permanent vision loss. The management vary significantly among different centers. Options of local corticosteroids included periocular or intraocular injections of TA and intraocular sustained-release glucocorticoid implants ${ }^{11,19}$. Of interest is the POINT trial which compared the effectiveness of 3 treatment modalities of local corticosteroids in UME, in particular periocular injections of 40mg TA (periorbital floor or posterior subTenon's approach), intraocular injections of $4 \mathrm{mg}$ TA and a $0.7 \mathrm{mg}$ dexamethasone intravitreal implant ${ }^{15}$. The results showed that all treatment groups had clinically meaningful reductions in central subretinal thickness compared with baseline ${ }^{15}$. However, subconjunctival injections of TA have rarely been reported $^{14-16}$.

\section{Regarding CMT}

In the first month after injection of $20 \mathrm{mg} \mathrm{TA}, 62 / 71$ eyes (87.32\%) showed a reduction in CMT with 59/71 eyes $(83.09 \%)$ by at least $20 \%$, which is very close to the overall response rate $(88 \%)$ observed in a previous study ${ }^{20}$ aiming to compare sub-tenon TA, intravitreal TA and intravitreal dexamethasone implants. Other studies, however, revealed lower levels of effectiveness. Bae and colleagues ${ }^{21}$ reported that $53.1 \%$ of the eyes treated with peribulbar injections of $40 \mathrm{mg}$ TA showed reduction in CMT after 1 month. Henry A. Leder et al. ${ }^{22}$ observed that UME was clinically resolved in $53 \%$ and $57 \%$ of treated eyes 1 and 3 months respectively after a single posterior-subtenon TA $(40 \mathrm{mg})$ injection. Furthermore, CMT reduction was observed only in $23 \%$ eyes 2 month after a periocular injections of $40 \mathrm{mg} \mathrm{TA}^{11}$.

\section{Regarding relapse}

As presented previously, twenty-two $(22 / 83,26.51 \%)$ eyes underwent relapse of UME within 6 months. Among these eyes, 5 (22.73\%), 7 (31.82\%) and 10 (45.45\%) eyes relapsed less than 2 months, 2 to 3 months, and 3 to 6 months after the injection, respectively. Some cases are worthy of note. In one patient, the first injection resulted in resolution of UME for 6 months, however, but the therapeutic effect of the second injection given 1.5 years later lasted only 2 months. Another patient received 7 injections with good responsiveness observed every time in a 10-year follow up period, and the longest resolution lasted for more than 6 months.

\section{Regarding IOP}

An elevated IOP was observed in 21/83 eyes (25.30\%) in our study. However, Byun et al. ${ }^{23}$ reported that 18 eyes (11.3\%) required glaucoma medications after a posterior-subtenon injection. Another study 
reported that $34.9 \%$ of the patients after a posterior-subtenon injection had elevated IOPs, and $4.7 \%$ of the patients needed trabeculectomy ultimately ${ }^{24}$.

Anterior subtenon injection of TA was found to be 2.4 times more likely $(95 \% \mathrm{Cl}, 1.02-5.9)$ to cause elevated IOPs than posterior subtenon injection ${ }^{25}$, which could be explained by the notion that a higher aqueous level of TA is associated with a higher incidence of IOP elevation. However, our data showed a similar rate of IOP elevation as compared to posterior subtenon injection. In addition, Elevated IOP was observed mainly (16/21 eyes) within the first 2 months after the injection in our study. However, 5 eyes develop IOP rise later than 2 months with the latest observed up to 15 weeks after the injection. While IOP-lowering eye drops were sufficient for majority of the patients, 7 eyes (7 patients) underwent surgical removal of the subconjunctival TA deposit and IOP returned to normal within 1 month after the surgery.

Subconjunctival hemorrhage is also a well-known but trivial side effect. Other reported side effects ${ }^{16}$ of subconjunctival TA such as conjunctival ulceration ${ }^{26}$, ischemia, necrosis ${ }^{27}$ and infectious scleritis, were not observed in our patients.

From our point of view, subconjunctival injection of TA (20mg) has several advantages over other periocular injections. It is technically an easier procedure and could be safely performed in the outpatient clinic; Even though it may be more likely to cause IOP elevation, topical IOP-lowering agents are usually sufficient to control the IOP, and surgical removal of subconjunctival TA deposits is easy and effective when intractable IOP elevation occurs.

There are some limitations for our study, including inhomogeneity of the included patients, inevitable biases, missing data and the different follow-up intervals among different patients due to the retrospective nature of the study.

\section{Conclusion}

In conclusion, subconjunctival TA injections appear to be safe and effective for UME. Increased IOP is a concern but could be well controlled by IOP-lowering eye drops and surgical removal of TA deposit when necessary.

\section{List Of Abbreviations}

TA : triamcinolone acetonide

UME: uveitic macular edema

OCT : optical coherence tomography

BCVA : best corrected visual acuity

IOP : intraocular pressure 
$\log M A R$ : logarithm of the minimal angle of resolution

\section{Declarations}

- Ethics approval and consent to participate: Yes

The study followed the tenets of the Declaration of Helsinki and was approved by the Ethics Committee of Peking Union Medical College Hospital. Written informed consent was obtained from all participants before the subconjunctival TA injection(s) and any other invasive procedures/examinations. While the participants are children (under 16 years old), the written informed consent was obtained from their parents.

- Consent for publication : Not applicable

- Availability of data and materials : We have uploaded an dataset of the 68 cases included IOP and CMT data.

- Competing interests: The authors declare that they have no competing interests.

- Funding: Not applicable

- Authors' contributions :

$Y Q, X L$ and $A L$ collected the data, while $Y Q$ wrote this article. $C Z, F G$ and $M Z$ reviewed, edited and reviesed the manuscript. All authors read and approved the manuscript.

- Acknowledgements: Not applicable

\section{References}

1. Accorinti M, Okada AA, Smith JR, Gilardi M. Epidemiology of Macular Edema in Uveitis. Ocul Immunol Inflamm. 2019;27:169-180.

2. Okhravi N, Lightman S. Cystoid macular edema in uveitis. Ocul Immunol Inflamm. 2003;11:29-38.

3. Lardenoye CW, van Kooij B, Rothova A. Impact of macular edema on visual acuity in uveitis. Ophthalmology. 2006;113:1446-9.

4. Multicenter Uveitis Steroid Treatment Trial Research G, Kempen JH, Altaweel MM, Holbrook JT, Jabs DA, Sugar EA. The multicenter uveitis steroid treatment trial: rationale, design, and baseline characteristics. Am J Ophthalmol. 2010;149:550-561 e10.

5. Symes RJ, Forooghian F. Topical difluprednate monotherapy for uveitic macular edema. Can J Ophthalmol. 2016;51:47-9.

6. Tallouzi MO, Moore DJ, Calvert M, Murray PI, Bucknall N, Denniston AK. The effectiveness of pharmacological agents for the treatment of uveitic macular oedema (UMO): a systematic review protocol. Syst Rev. 2016;5:29. 
7. Lindholm JM, Taipale C, Ylinen P, Tuuminen R. Perioperative subconjunctival triamcinolone acetonide injection for prevention of inflammation and macular oedema after cataract surgery. Acta Ophthalmol. 2020;98:36-42.

8. Sreekantam S, Macdonald T, Keane PA, Sim DA, Murray PI, Denniston AK. Quantitative analysis of vitreous inflammation using optical coherence tomography in patients receiving sub-Tenon's triamcinolone acetonide for uveitic cystoid macular oedema. Br J Ophthalmol. 2017;101:175-179.

9. Levin MH, Pistilli M, Daniel E, et al. Incidence of visual improvement in uveitis cases with visual impairment caused by macular edema. Ophthalmology. 2014;121:588-95 e1.

10. Tranos PG, Tsaousis KT, Vakalis AN, Asteriades S, Pavesio CE. Long-term follow-up of inflammatory cystoid macular edema. Retina. 2012;32:1624-8.

11. Choudhry S, Ghosh S. Intravitreal and posterior subtenon triamcinolone acetonide in idiopathic bilateral uveitic macular oedema. Clin Exp Ophthalmol. 2007;35:713-8.

12. Sen HN, Vitale S, Gangaputra SS, et al. Periocular corticosteroid injections in uveitis: effects and complications. Ophthalmology. 2014;121:2275-86.

13. Steeples LR, Anand N, Moraji J, Jones NP. Clinical Outcomes of Intravitreal Preservative-Free Triamcinolone Preparation (Triesence(R)) for Cystoid Macular Oedema and Inflammation in Patients with Uveitis. Ocul Immunol Inflamm. 2018;26:997-1004.

14. Liu $X$, Wang M, Zhao $C$, Gao F, Zhang M. [The efficacy and safety of subconjunctival triamcinolone acetonide injections in treatment of uveitic macular edema]. Zhonghua Yan Ke Za Zhi. 2015;51:7348.

15. Thorne JE, Sugar EA, Holbrook JT, et al. Periocular Triamcinolone vs. Intravitreal Triamcinolone vs. Intravitreal Dexamethasone Implant for the Treatment of Uveitic Macular Edema: The PeriOcular vs. INTravitreal corticosteroids for uveitic macular edema (POINT) Trial. Ophthalmology. 2019;126:283295.

16. Athanasiadis $Y$, Tsatsos M, Sharma A, Hossain P. Subconjunctival triamcinolone acetonide in the management of ocular inflammatory disease. J Ocul Pharmacol Ther. 2013;29:516-22.

17. Jones NP. The Manchester Uveitis Clinic: the first 3000 patients-epidemiology and casemix. Ocul Immunol Inflamm. 2015;23:118-26.

18. Lasave AF, Schlaen A, Zeballos DG, et al. Twenty-Four Months Follow-Up of Intravitreal Bevacizumab Injection Versus Intravitreal Triamcinolone Acetonide Injection for the Management of Persistent Non-Infectious Uveitic Cystoid Macular Edema. Ocul Immunol Inflamm. 2019;27:294-302.

19. Roesel M, Gutfleisch M, Heinz C, Heimes B, Zurek-Imhoff B, Heiligenhaus A. Intravitreal and orbital floor triamcinolone acetonide injections in noninfectious uveitis: a comparative study. Ophthalmic Res. 2009;42:81-6.

20. Carbonniere C, Couret C, Bleriot A, et al. [Treatment of macular edema: Comparison of efficacy and tolerability of subconjunctival triamcinolone injections, sub-tenon's triamcinolone injections and intravitreal dexamethasone implant]. J Fr Ophtalmol. 2017;40:177-186. 
21. Bae JH, Lee CS, Lee SC. Efficacy and safety of intravitreal bevacizumab compared with intravitreal and posterior sub-tenon triamcinolone acetonide for treatment of uveitic cystoid macular edema. Retina. 2011;31:111-8.

22. Leder HA, Jabs DA, Galor A, Dunn JP, Thorne JE. Periocular triamcinolone acetonide injections for cystoid macular edema complicating noninfectious uveitis. Am J Ophthalmol. 2011;152:441-448 e2.

23. Byun YS, Park YH. Complications and safety profile of posterior subtenon injection of triamcinolone acetonide. J Ocul Pharmacol Ther. 2009;25:159-62.

24. Jea SY, Byon IS, Oum BS. Triamcinolone-induced intraocular pressure elevation: intravitreal injection for macular edema and posterior subtenon injection for uveitis. Korean J Ophthalmol. 2006;20:99103.

25. Liu X, Li Y, Zhang Y, et al. Comparison of intraocular pressure elevation after anterior versus posterior subtenon triamcinolone acetonide acetate injection: a retrospective study. Retina. 2012;32:1838-43.

26. Agrawal S, Agrawal J, Agrawal TP. Conjunctival ulceration following triamcinolone injection. American Journal of Ophthalmology. 2003;136:539-540.

27. Ying-Jiun C, Chee-Kuen W, Shatriah I. Conjunctival necrosis following a subconjunctival injection of triamcinolone acetonide in a child. Middle East Afr J Ophthalmol. 2015;22:125-8.

\section{Table}

TABLE 1. Demographic features of the patients. 
Uveitis diagnosis

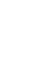

Sex

Age

Lens condition

Periocular steroid injection times

bil

\begin{tabular}{|c|c|c|c|}
\hline 7times & 1 & patient & $1.47 \%$ \\
\hline 6times & 1 & patient & $1.47 \%$ \\
\hline 5times & 1 & patient & $1.47 \%$ \\
\hline 4times & 5 & patients & $7.35 \%$ \\
\hline 3times & 7 & patients & $10.29 \%$ \\
\hline 2times & 15 & patients & $22.06 \%$ \\
\hline 1times & 38 & patients & $55.88 \%$ \\
\hline
\end{tabular}

Systemic Therapy bilateral 15 patients $22.06 \%$

$48.53 \%$ Idiopathic

$27.94 \%$ Vogt-Koyanagi-Harada disease

4.41\% Tuberculosis-associated

4.41\% JIA-associated

$2.94 \%$ Sarcoidosis

2.94\% Bechçet disease

$1.47 \%$ HLA-B27 associated

$7.35 \%$ Other

75\% Female

25\%. Male

Range from 11 to 78

Mean $\pm S D \quad 49.2 \pm 14.1$

$51.47 \%$ no cataract

$26.57 \%$ already with complicated cataract

$22.06 \% \quad$ IOL eyes

unilateral 53 patients $77.94 \%$

$64.7 \%$ of all patients

Page 10/13 
$8.8 \%$ prednisolone alone

$36.8 \%$ prednisolone +1 immunosuppressant

$11.8 \%$ prednisolone +2 immunosuppressant

$4.4 \% 1$ immunosuppressant

$2.9 \% 2$ immunosuppressant

\section{Figures}

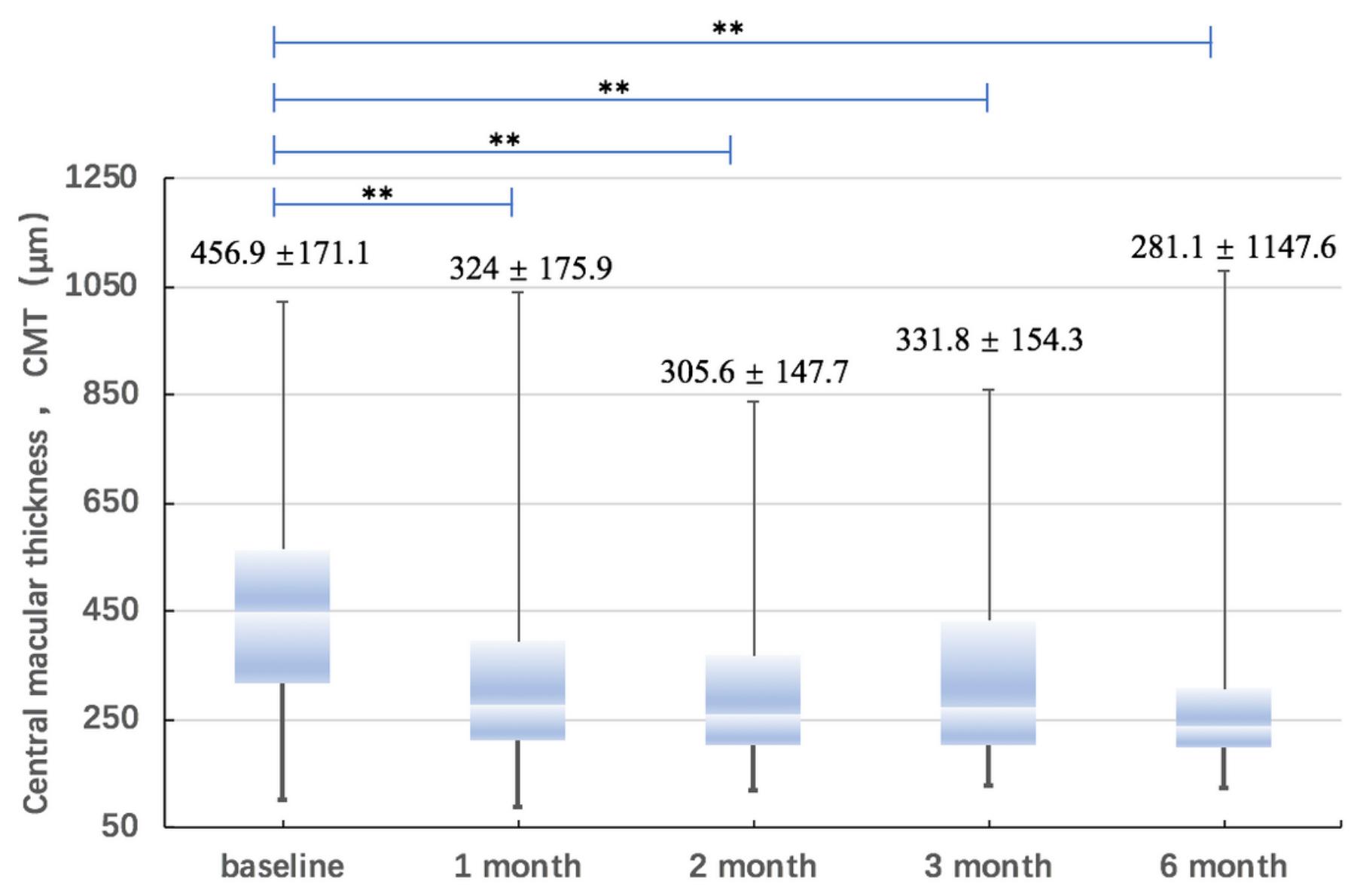

Figure 1

CMT changes after the treatment with subconjunctival injection of TA CMT: Central macular thickness.

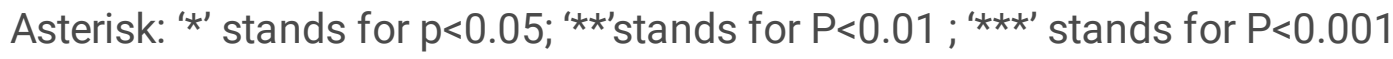




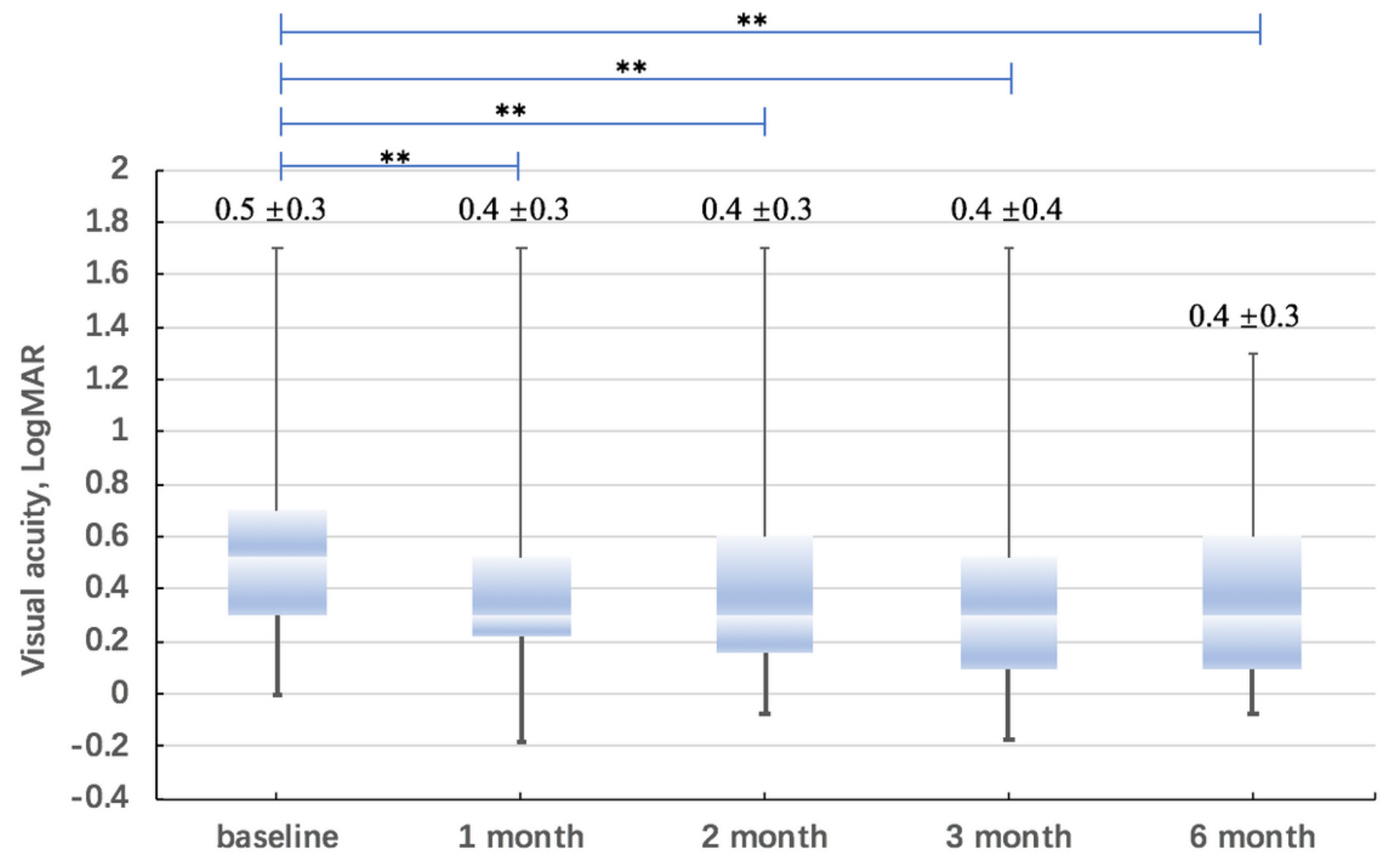

Figure 2

Mean baseline visual acuity and the changes over time. VA: Visual acuity; logMAR : logarithm of the

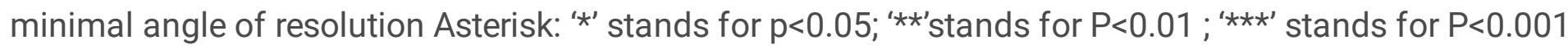




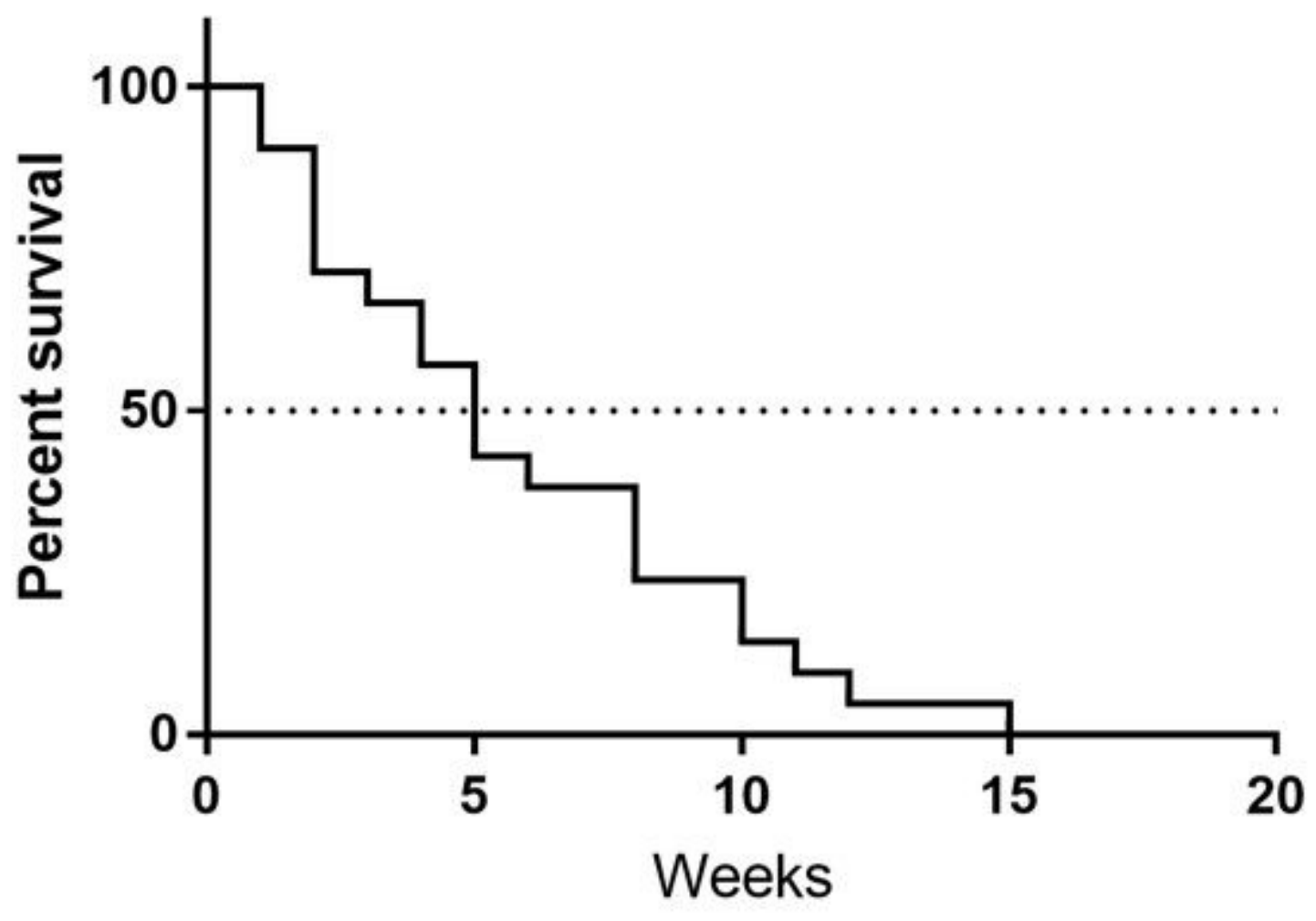

Figure 3

Kaplan-Meier survival analysis for patients with IOP $>21 \mathrm{mmHg}$

\section{Supplementary Files}

This is a list of supplementary files associated with this preprint. Click to download.

- Additionalfile1originaldataset.xlsx 